\title{
Gestion des ressources naturelles : la régénération des bourgoutières dans la boucle du Niger au Mali
}

\author{
J.-M. Bonis Charancle ${ }^{1}$
}

BONIS CHARANCLE' (J-M.). Gestion des ressources naturelles : la régénération des bourgoutières dans la boucle du Niger au Mali. Revue Élev. Méd. vét. Pays trop., 1994, 47 (4) : 425-434

La boucle du Niger au Mali est une région où l'on observe de nombreux phénomènes de désertification depuis le début des années 1970 . Ainsi, les bourgoutières, riches prairies inondables, ont quasiment disparu du Cercle de Tombouctou entre 1970 et 1985. L'article décrit les efforts des populations de cette division administrative et ceux de Vétérinaires Sans Frontières pour s'opposer à la dégradation des bourgoutières. Il aborde les aspects technique, organisationnel et économique du "programme bourgou", qui a abouti à la régénération de plus de 2000 ha, puis tire les lecons de cette expérience. D'un point de vue environnemental, un équilibre fragile a été atteint mais qui serait remis en cause par une nouvelle dégradation climatique. L'utilisation abusive par les troupeaux se révèle être plus un facteur additionnel de dégradation qu'un facteur primaire. Du point de vue de l'organisation des bénéficiaires, le travail de régénération a eu pour conséquence fondamentale la parcellisation des anciennes vaines pâtures et leur attribution à des individus responsables de leur entretien, dans le cadre de règles collectivement fixées. Mais, au plan du droit foncier, l'auteur estime que les outils nécessaires à la gestion des conflits sur les ressources naturelles et à la défense des promoteurs d'activités de régénération ne sont pas encore en place.

Mots clés : Bourgoutière - Echinochloa - Ressource naturelle - Dégradation - Régénération - Pâturage - Elevage - Désertification Environnement - Population rurale - Politique foncière - Facteur climatique - Fleuve Niger - Mali - Sahel.

\section{INTRODUCTION}

Le premier contact de Vétérinaires Sans Frontières (VSF) en sa qualité d'ONG avec les populations du Cercle de Tombouctou date de 1984 avec un programme d'urgence "Viande séchée" dont le but est d'aider les éleveurs à valoriser, autant que possible, leur cheptel condamné par la sécheresse. A la suite de cette intervention, VSF est de nouveau sollicité pour concevoir et exécuter un projet de relance des activités d'élevage dans la phase de posturgence qui s'amorce. La première activité identifiée est la régénération des bourgoutières, pâturages cruciaux qui se sont dégradés entre 1970 et 1985 . Trois années seront consacrées à cette unique activité : 1985, 1986, 1987, au cours desquelles près de 1000 ha de pâturages seront régénérés avec 40 collectivités.

VSF continuera ensuite à travailler sur des aspects de gestion sans s'impliquer dans les activités de régénération, bien maîtrisées par les populations. De 1988 à 1993

1. Vétérinaires Sans Frontières (VSF), Espace Rhône-Alpes Coopération, 14 ave Berthelot, 69361 Lyon Cedex 07, France.

Reçu le 23.2.1994, accepté le 7.3.1995. le projet VSF-Tombouctou diversifiera ses activités, avec pour objectif d'appuyer techniquement et financièrement les tentatives des producteurs pour surmonter les nouvelles contraintes, essentiellement dues au milieu, la plus courante étant l'intensification d'activités intégrées d'agriculture et d'élevage sur la frange du fleuve Niger. Seront développés dans ce contexte trois volets : régénération et gestion des ressources naturelles, intégration agricultureélevage et formation. Profitant de la présence continue d'équipes VSF à Tombouctou depuis 1984, cet article fait le bilan de l'activité de "régénération" cinq ans après la clôture de ce volet.

\section{DONNÉES SUR LE BOURGOU ET SUR LE PROGRAMME BOURGOUTIÈRES}

\section{Données écologiques}

Les bourgoutières sont des prairies aquatiques inondées (2) par la crue du Niger dans lesquelles pousse un pâturage naturel de graminées largement dominé par le bourgou (Echinochloa stagnina). La montée des eaux a lieu entre juillet et novembre. Elle est donc concomitante de la saison des pluies (juillet à septembre). Le fleuve sort alors de son lit mineur pour envahir de nombreux bras et mares. Le retrait débute au mois de décembre et est rapide jusqu'au mois de février (cette période correspond à la saison sèche froide). Le pâturage est accessible au bétail à compter du mois de février et jusqu'à l'arrivée de la crue suivante, soit durant une période de six mois.

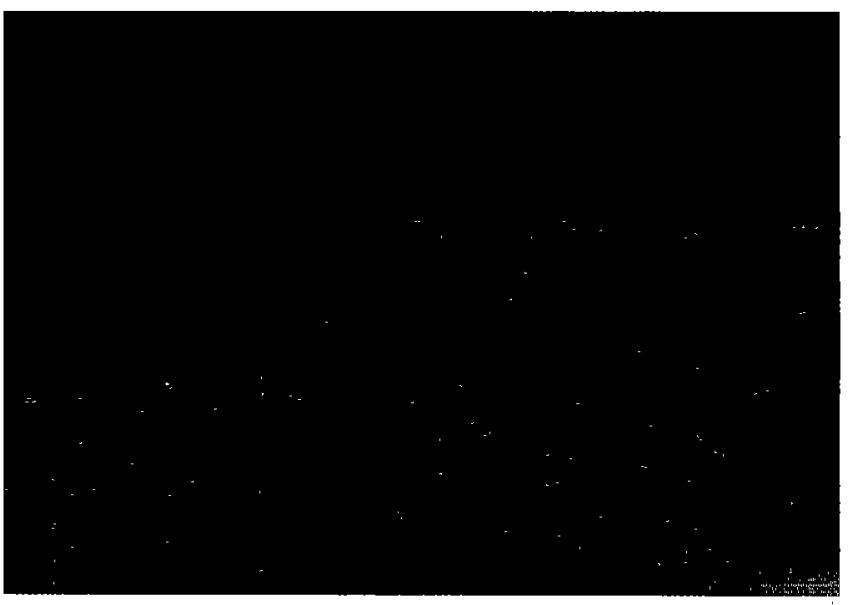

Photo 1 ; Bourgoutière, région de Mopti, Mali (cliché G. BOUDET). 
Le bourgou est donc un pâturage de soudure disponible essentiellement durant la fin de la saison sèche froide et durant la saison sèche chaude. Or, les bourgoutières sont en voie de disparition par la conjonction de différents facteurs :

- baisse de la pluviosité : la sécheresse empêche la germination et la repousse du bourgou avant la crue : s'il est trop petit et fragile, il sera submergé et pourrira. La pluviosité à Tombouctou est passée d'une moyenne de $191 \mathrm{~mm}$ pour la période 1922-1987 à $116 \mathrm{~mm}$ pour la période 1981-1987 (1) ;

- baisse des crues : la faiblesse de la crue réduit la surface et la productivité des bourgoutières. La cote maximale moyenne du fleuve durant la période 1924-1985 est de $559 \mathrm{~cm}$ à Diré, atteinte durant la troisième décade de décembre. Pour la période 1981-1987 elle est de $428 \mathrm{~cm}$, atteinte la première ou la seconde décade de novembre. La crue a donc perdu $1,3 \mathrm{~m}$ en hauteur et 45 jours en durée (1). Devenant accessible plus tôt, la bourgoutière perd peu à peu son caractère de pâturage de soudure ;

- les bourgoutières sont surpâturées depuis 1970. Les éleveurs tentent de conserver le maximum de bétail au cours des mauvaises périodes, dépassant ainsi les capacités de charge des pâturages. La régulation du nombre d'animaux se fait alors par chocs successifs, lorsque les ressources végétales deviennent insuffisantes;

- depuis 20 ans, l'Etat, au moyen de grands projets, a donné la priorité au développement de la riziculture au nom de l'objectif de l'autosuffisance. Les bourgoutières ont donc souvent été négligées et ont dû céder en partie la place au riz.

Le bilan conjugué de tous ces facteurs de dégradation est lourd : avant 1970 , les bourgoutières du Cercle de Tombouctou couvraient une surface estimée entre 8000 et 10000 ha. En 1985, il ne restait que quelques dizaines d'hectares disséminés.

La disparition du bourgou a eu d'autres conséquences car, cette riche unité écologique avait de multiples fonctions (figure 1).

\section{Situation foncière historique}

La première tentative d'organisation foncière autour des bourgoutières remonte à la Dina de Cheikou Amadou. Mais c'est surtout la région de Mopti, sous influence directe Peule, qui fut concernée avec la mise en place des Dioros, propriétaires-gérants, organisation qui perdure encore aujourd'hui. Certaines collectivités de Tombouctou, probablement celles qui reconnaissaient la suprématie des Peul, ont des documents de cette époque, les tariks, qui leur reconnaissent des droits fonciers sur des terres.

La deuxième tentative d'organisation foncière autour des terres de la frange fluviale date de la colonisation. Des conventions furent rédigées, principalement entre 1920 et 1940 , attribuant le droit d'usage de bourgoutières précisément désignées à des groupes identifiés. Ces conventions essayaient de formaliser l'utilisation visible des
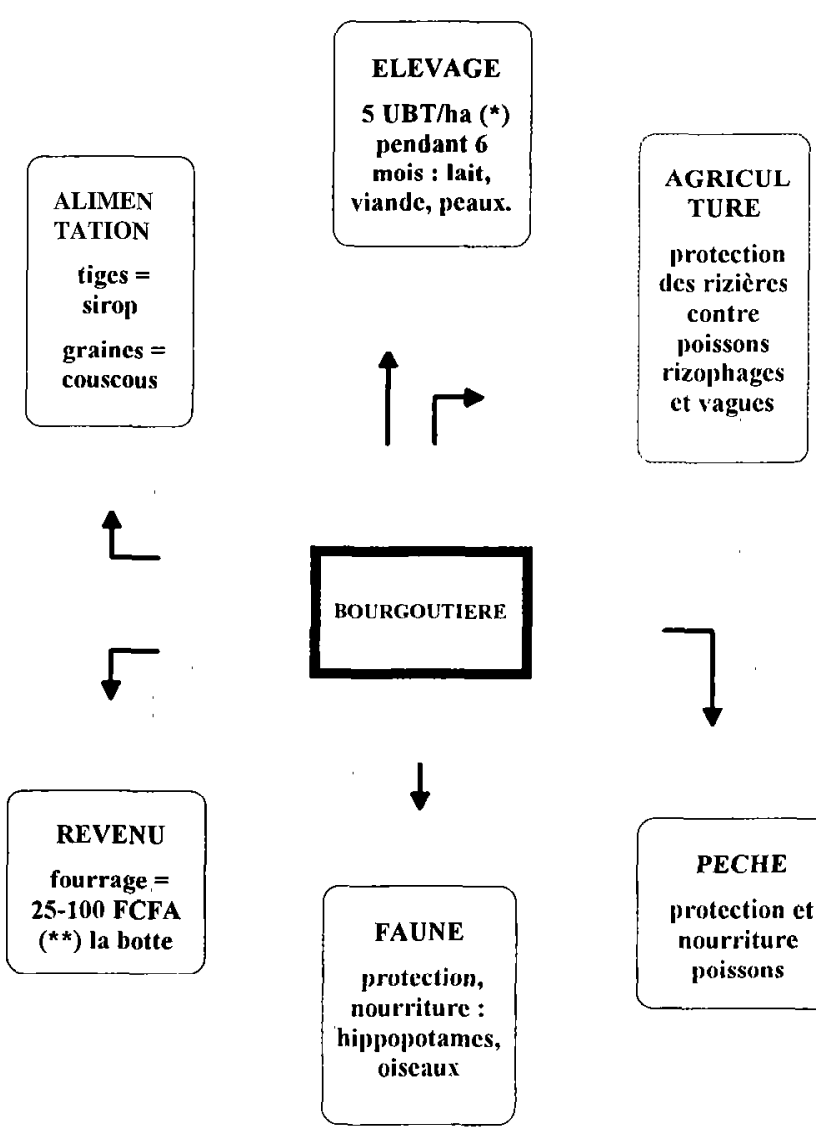

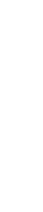




\section{Données agronomiques sur le bourgou}

Le bourgou est une graminée pluriannuelle se développant avec la crue dans les plaines inondables du Niger: Echinochloa stagnina (bourgou rouge) et Echinochloa pyramidalis (bourgou blanc) sont les deux espèces rencontrées le plus fréquemment (3). La reprise annuelle du bourgou est due à la germination des semences, ainsi qu'au redémarrage de pieds pérennes. Le bourgou se rencontre dans des zones où la lame d'eau est comprise entre 1 et 4 mètres.

La production atteint régulièrement $10 \mathrm{t}$ utiles de matière sèche $(\mathrm{MS}) /$ ha (production totale $=$ utile + pertes par piétinement, refus ou résidus de récolte ).

Voici la valeur alimentaire $(4,7)$ pour les trois utilisations possibles:

- lors du pâturage direct à la décrue, l'apport d'1 kg de MS est de l'ordre de 0,55 unité fourragère (UF) et $20 \mathrm{~g}$ de matières azotées digestibles (MAD) ;

- lors du pâturage sur repousses après fauche ou première pâture, l'apport d' $1 \mathrm{~kg}$ de MS est de l'ordre de 0,45 UF et $65 \mathrm{~g}$ de MAD;

- le fourrage récolté apporte 0,5 UF et $35 \mathrm{~g}$ de MAD. La récolte concerne 50 à 75 p. 100 de la biomasse, le reste étant utilisé en pâture après fauchage.

Stocké au soleil et au vent, le fourrage perd de sa valeur avec le temps. Le déficit en MAD observé lors de la pâture directe, ainsi qu'un coefficient de digestibilité bas (55 p. 100 ) du fait d'un taux de cellulose élevé (30 p. 100) suggèrent qu'il est raisonnable de retenir une capacité de charge moyenne de 5 UBT/ha pendant la période d'utilisation de six mois.

TABLEAU I Charge théorique d'une bourgoutière en fonction de la matière sèche, des UF ou des MAD.

\begin{tabular}{|l|c|c|}
\hline $\begin{array}{l}\text { Besoins d'entretien } \\
\text { pour une UBT }\end{array}$ & $\begin{array}{c}\text { Apport d'un ha } \\
\text { en pâturage de décrue }\end{array}$ & $\begin{array}{c}\text { Charge possible durant } \\
\text { la période d'utilisation } \\
\text { de } 6 \text { mois }\end{array}$ \\
\hline $6,25 \mathrm{~kg} \mathrm{MS} /$ jour & 10000 & $8,9 \mathrm{UBT}$ \\
\hline $2,3 \mathrm{UF} /$ jour & 5500 & $13,3 \mathrm{UBT}$ \\
\hline $150 \mathrm{~g}$ MAD/jour & 200000 & $7,4 \mathrm{UBT}$ \\
\hline
\end{tabular}

\section{Technique de régénération}

La technique de régénération retenue est le repiquage de plants ou boutures en période de crue. Cette technique (8) comporte trois phases:

\section{Approvisionnement en plants ou boutures}

Les éclats de souche et boutures sont extraits des bourgoutières existantes à raison d'une surface de prélèvement de $500 \mathrm{~m}^{2}$ pour repiquer un hectare (rapport de
1/20). Les plants sont obtenus en pépinière, du type pépinière de riz, mais contrairement au riz, le plant de bourgou ne doit pas être enlevé de la pépinière avant le tallage. Le semis se fait en poquets. L'arrosage manuel nécessite par jour $30 \mathrm{l} / \mathrm{m}^{2}$. Le temps de travail est de deux heures par jour pour une pépinière de 16 planches de $2 \mathrm{~m}^{2}$. Un arrosage par motopompe peut être utile en cas de pépinière de grande taille. La germination a lieu sept jours après le semis. Le tallage commence à trois semaines, les boutures sont prêtes après deux ou trois mois (taille de 40 à $80 \mathrm{~cm}$ ). La pépinière nécessite un gardiennage pour être protégée des prédateurs : cheptel, hippopotames. Une planche de $2 \mathrm{~m}^{2}$ de pépinière produit environ 1000 boutures.

\section{Repiquage}

Le repiquage peut se faire à trois périodes : au moment des pluies, au moment de la montée des eaux, au moment de la décrue. Le repiquage à la montée des eaux (août à septembre), dans la zone de Tombouctou, est préconisé comme le plus sûr. Le repiquage est effectué à la main ou avec un bâton fourchu quand l'eau est un peu haute : le plant ou la bouture doit avoir deux nœuds émergés avec des feuilles sinon il (elle) risque l'asphyxie. La reprise a lieu en sept jours, durant lesquels il est nécessaire que la montée des eaux ne soit pas trop rapide pour éviter les risques de submersion. On repique tous les $80 \mathrm{~cm}$ soit environ 16000 boutures pour un hectare. II faut donc une pépinière de $32 \mathrm{~m}^{2}$ (16 planches de $2 \mathrm{~m}^{2}$ ) pour repiquer un hectare et 10 hommes/jour pour ce travail.

\section{Protection et exploitation}

La bourgoutière doit être gardée pendant deux ans afin d'éviter des dégradations irréversibles en période de croissance. Pour une bourgoutière ayant une lame d'eau de $1,5 \mathrm{~m}$, la productivité atteint 1,5 tha de MS la première année, $10 \mathrm{t} /$ ha la deuxième ánnée et se stabilise autour de 15 t/ha la troisième année. En saison sèche, le plant est pérenne. II se couche sur le sol et marcotte. Le piétinement par le bétail renforce ce marcottage mais le pâturage par le bétail ne doit être ni excessif ni trop tardif, sinon la repousse sera insuffisante à l'arrivée de la crue. Une bonne conduite du troupeau peut permettre deux pâtures : l'une immédiate au retrait des eaux, l'autre après repousse.

\section{Organisation des groupes concernés par la régénération (8)}

\section{Choix des plaines à régénérer}

Dans la plupart des cas, le choix des plaines s'est déroulé suivant le processus ci-dessous:

- désignation d'un site par le conseil de la collectivité. Dans la région de Tombouctou, l'autorité traditionnelle est constituée d'un chef reconnu par l'administration, entouré de conseillers ; 
- vérification du respect de critères techniques par l'équipe VSF ;

- demande d'accord administratif se traduisant par l'inscription du site à régénérer dans le programme d'activité du comité local de développement en cas d'acceptation.

Dans certains cas, le rôle de l'administration a été plus actif : rôle de médiation entre les collectivités pour aboutir à des compromis sur des terres litigieuses ; attribution d'office de parcelles à des collectivités n'ayant pas d'accès traditionnel au fleuve.

\section{Organisation pour la régénération}

VSF et l'administration ont suscité la création de Comités de gestion des bourgoutières comprenant le conseil villageois, un représentant du service de l'Elevage et le représentant de VSF. A la demande de ce dernier, les conseils des collectivités ont procédé à un parcellaire des plaines.

La répartition ne s'est pas faite de manière égalitaire et en général, les chefs de village ou de fraction et leurs conseillers ont bénéficié de plusieurs parcelles.

Le repiquage est individuel et progressif, au fur et à mesure de la montée des eaux. Ce travail a été soutenu par des distributions de rations alimentaires (food for work $^{\star}$, en quantités décroissantes de 1985 à 1987. Chaque plaine a été confiée a un gardien rétribué pendant un an par VSF. Les années suivantes, les exploitants décident seuls de régénérer d'autres bourgoutières individuellement ou collectivement et se procurent les plants ou boutures par leurs propres moyens. Le projet continue à assurer le conseil et le suivi.

\section{Organisation pour l'exploitation}

La fauche est pratiquée individuellement lors du retrait des eaux. Elle concerne les feuilles, les tiges émergées et une partie des tiges immergées. Entre 50 et 75 p. 100 de la biomasse sont retirés par la fauche. Le fourrage sèche à proximité de la bourgoutière puis est stocké sur les toits des cases ou dans les arbres. La pâture a lieu collectivement, soit après la fauche, soit sur la bourgoutière non fauchée. La date d'entrée des animaux sur le pâturage est décidée par le Comité de gestion.

\section{Valeur économique du bourgou}

\section{Vente du fourrage}

II y a un important marché du fourrage de bourgou à Tombouctou qui approvisionne les citadins ayant des animaux de case. En 1987, la botte de bourgou (équivalente à $1,5 \mathrm{~kg}$ de MS) a vu son prix évoluer de $25 \mathrm{~F} \mathrm{CFA} \mathrm{en}$ janvier à $100 \mathrm{~F}$ CFA en juillet.

\footnotetext{
* Des rations alimentaires (food for work) ont été fournies par des agences des Nations unies (UNICEF, PAM), après constatation de l'incapacité physique des bénéficiaires du programme à réaliser les travaux nécessaires sans cet appui.
}

Chaque année, ce marché rapporte aux propriétaires, exploitants, transporteurs et vendeurs la somme de 30 millions de F CFA, soit l'équivalent du budget annuel familial de 120 familles de 10 personnes (le Cercle compte 100000 habitants). Un hectare de bourgou produit 10 tonnes de fourrage exploitable dont la valeur est de $250000 \mathrm{~F}$ CFA /ha à un prix moyen de $40 \mathrm{~F}$ CFA par botte.

Dans la pratique, on constate que la fauche ne concerne que 50 à 75 p. 100 de la production, le reste étant laissé pour la pâture. De plus, le marché est limité par l'effectif des animaux de case de Tombouctou. Les chiffres précédents indiquent que c'est la production de 120 ha qui est commercialisée, soit 5 à 10 p. 100 de la surface totale suivant les années.

\section{Exploitation directe par un troupeau de bovins}

Le calcul de la plus-value apportée par la régénération d'une bourgoutière est fait sur les bases suivantes:

- toute la production est attribuée à la régénération de la bourgoutière. On considère alors que le troupeau peut accomplir son cycle entier grâce à l'accès à une bourgoutière ;

- la charge supportée par un hectare pendant ses six mois d'exondation est de 5 UBT, soit 6,25 bovins.

Dans ce cas, le troupeau est stabilisé, c'est-à-dire qu'il n'augmente pas en poids et en effectif. Son taux d'exploitation net est de $10 \mathrm{p} .100$ et il comprend à tout moment 18 p. 100 de vaches traites donnant 0,8 I de lait par jour pour l'alimentation humaine.

La valeur de la production annuelle est la suivante

- vente d'animaux : 6,25 x 0,1 x 50000 F CFA = 31250 F CFA ;

- vente ou consommation de lait : $6,25 \times 0,18 \times$ 200 F CFA $\times 365$ j = 82125 F CFA.

Au total, la plus-value apportée par la régénération d'un hectare exploité en pâturage de décrue est de 113375 F CFA. La régénération est donc d'un grand rapport, tout en demandant peu d'entretien. On comprend que les exploitants se lancent seuls dans cette pratique.

\section{Coût de la régénération (8)}

Les coûts se subivisent comme suit :

- coût de l'équipc de projet : un volontaire VSF et le personnel national ;

- coût de la logistique ;

- coût de l'activité proprement dite : achat de semences, de boutures et de sacs pour leur transport, de matériel pour les pépinières, salaires des gardiens, etc. ; 
- coût de l'apport alimentaire d'organisations spécialisées (UNICEF, PAM) ${ }^{\star}$;

- coût de l'appui administratif et technique apporté par VSF.

En 1985, l'action test de régénération de bourgoutières à Hondo Bomo Koina et avec les Tell Emedess est revenue à 80000 F CFA/ha. En 1986 et 1987, ce coût a été réduit à $25000 \mathrm{~F}$ CFA/ha grâce à des économies d'échelle, à la diminution des actions food for work et à un approvisionnement en boutures moins onéreux ${ }^{\star \star}$ (tabl. II).

TABLEAU II Coûts de la régénération des bourgoutières du Cercle de Tombouctou.

\begin{tabular}{|c|c|c|c|}
\hline Année & ha régénérés & coût/ha (F CFA) & coût total (F CFA) \\
\hline 1985 & 80 & 80000 & 6400000 \\
\hline 1986 & 320 & 25000 & 8000000 \\
\hline 1987 & 400 & 25000 & 10000000 \\
\hline
\end{tabular}

\section{LES LEÇONS DE L'EXPÉRIENCE}

\section{Environnement}

L'analyse est basée sur la comparaison des courbes de surface des bourgoutières, de pluviosité, de hauteur de crue et d'effectifs de cheptel dans le Cercle de Tombouctou entre 1984 et 1992 (fig. 2, 3, 4) (tabl. III). Deux constatations montrent une sorte d'appropriation technique par les populations locales:

- les surfaces ont continué à augmenter après que le projet ait cessé d'être impliqué dans les activités de régénération proprement dite (c'est-à-dire après 1987) ;

- les surfaces perdues en 1990 ont été récupérées dès 1991, leur diminution en 1990 étant due à un démarrage de crue brutale non précédé de pluies.

Aussi, dans les limites climatiques actuellement connues, la régénération est une pratique soutenue et durable.

En 1970, le Cercle de Tombouctou comptait entre 8000 et 10000 ha de bourgoutières. Ce chiffre ne pourra plus être atteint en raison de la diminution de la hauteur de crue. La surface maximale possible dans les conditions actuelles est comprise entre 3000 et 3500 ha. En réduisant l'inondation, la dégradation climatique a diminué de plus de 50 p. 100 les terres à bourgoutières. II n'apparaît pas cependant de corrélation évidente entre les facteurs

\footnotetext{
* Le food for work a été donné à VSF, mais on a pris en compte sa valeur financière pour calculer le coût de la régénération.

** (Production en pépinières dans chaque collectivité, fourniture de "boutures de remboursement" par les collectivités déjà engagées dans le programme, achat de boutures sur des sites moins éloignés.)
}

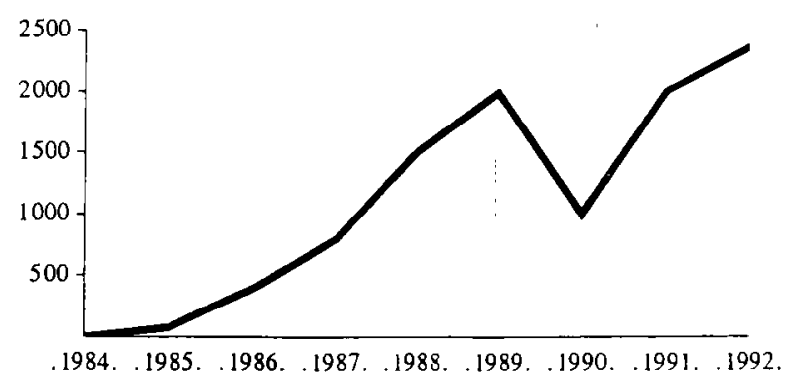

Bourgou. ha

Figure 2 : Evolution de la surface des bourgoutières dans le Cercle de Tombouctou entre 1984 et 1992.
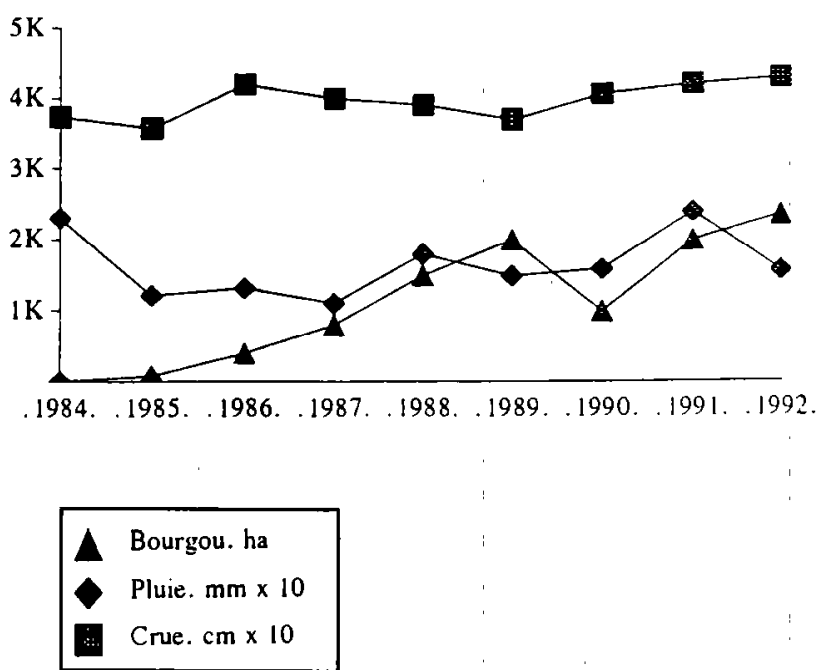

Figure 3 : Comparaison de l'évolution des surfaces de bourgoutières avec les facteurs hydriques: pluie et crue.

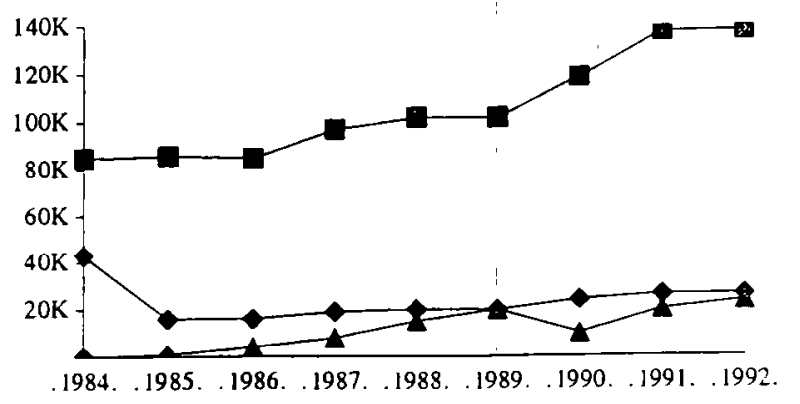

Figure 4 : Comparaison de l'évolution des surfaces de bourgoutières avec les effectifs du bétail. 
TABLEAU III Principales données sur les bourgoutières du Cercle de Tombouctou

\begin{tabular}{|c|c|c|c|c|c|c|c|c|c|}
\hline & 1984 & 1985 & 1986 & 1987 & 1988 & 1989 & 1990 & 1991 & 1992 \\
\hline $\begin{array}{l}\text { Surfaces de bourgoutières dans } \\
\text { le cercle de Tombouctou (ha) }\end{array}$ & 0 & 80 & 400 & 800 & 1500 & 2000 & 1000 & 2000 & 2350 \\
\hline Pluviosité. Tombouctou (mm) & 231 & 122 & 1332 & 111 & 180 & 150 & 160 & 240 & 157 \\
\hline Hauteur crue. Diré $(\mathrm{cm})$ & 374 & 359 & 420 & 400 & 390 & 370 & 406 & 420 & 428 \\
\hline $\begin{array}{l}\text { Effectif des bovins du cercle de } \\
\text { Tombouctou }\end{array}$ & 43000 & 16000 & 16000 & 19000 & 20000 & 20000 & 24000 & 26000 & 26000 \\
\hline $\begin{array}{l}\text { Effectif des petits ruminants du } \\
\text { du cercle de Tombouctou }\end{array}$ & 85000 & 86000 & 85000 & 97000 & 102000 & 102000 & 119000 & 138000 & 138000 \\
\hline
\end{tabular}

hydriques et les surfaces, sur la période considérée (fig. 3). Néanmoins, sur de plus longues périodes, les facteurs sont corrélés (1).

Comme pour la plupart des productions du Sahel, les facteurs hydriques interviennent plus par leur régularité que par leur quantité. Ainsi une crue forte au début $(10 \mathrm{~cm} / \mathrm{jour})$ ou l'absence de pluies au mois de juillet auront des effets désastreux sur les bourgoutières. Un nouvel aléa climatique pourrait donc remettre en cause la situation actuelle. Entre 1987 et 1991, on observe une superposition de la courbe des surfaces avec celle de la pluviosité. Cette superposition cesse en 1992, la baisse de la pluviosité ne s'accompagnant pas d'une diminution des surfaces (fig. 3). Ceci pourrait signifier un degré de maîtrise technique des populations, leur permettant un certain niveau d'affranchissement par rapport aux conditions climatiques.

Lorsque l'on compare les effectifs du bétail (fig. 4), on remarque que 1985-1992 est une période de recapitalisation. Entre 1987 et 1992 (phase post-projet), les effectifs croissent moins vite que les surfaces de bourgou (tabl. IV). Néanmoins, les surfaces de bourgou actuelles ne peuvent nourrir que 27 p. 100 du bétail du Cercle (2 350 ha x 5 UBT $=11750$, alors qu'il y a 43800 UBT dans le Cercle de Tombouctou). On est donc d'ores et déjà en présence d'une population animale qui, en cas de mauvaise année de pâturages dunaires, peut se concentrer sur les bourgoutières, au risque de leur dégradation.

Pour des raisons de survie à court terme, la stratégie des éleveurs est de reconstituer numériquement leur cheptel dès que les conditions deviennent un tant soit peu favorables. L'élevage dans ces zones fragiles peut alors devenir prédateur et facteur de désertification. Le croît du cheptel est surtout le fait des troupeaux des agriculteurs et des fonctionnaires. Pour subvenir à leurs besoins essentiels, les éleveurs traditionnels dont les activités sont moins diversifiées, sont contraints d'exploiter davantage leurs troupeaux.

En conclusion, on retiendra que le risque majeur pour les bourgoutières est d'abord d'ordre climatique. Les dégradations dues à l'élevage sont secondaires, localisées et récupérables par le travail et la volonté de l'homme.
TABLEAU IV Taux de croît (en p. 100) des surfaces de bourgoutières et des effectifs de bétail.

\begin{tabular}{|l|c|}
\hline & $1987-1992$ \\
\hline Surfaces de bourgou & +194 \\
\hline UBT du Cercle de Tombouctou & +40 \\
\hline Bovins du Cercle de Tombouctou & +37 \\
\hline Ovins du Cercle de Tombouctou & +33 \\
\hline Caprins du Cercle de Tombouctou & +50 \\
\hline
\end{tabular}

\section{Organisation des populations}

\section{Organisation moderne et traditionnelle - Evolution}

Dans les années 1970, l'organisation existante autour des bourgoutières est la suivante :

- l'ensemble des terres appartient à l'Etat qui ne reconnaît que des droits d'usage ;

- certaines collectivités ont des documents leur attribuant des droits d'usage sur des terres identifiées (tariks, conventions).

En général, l'Etat, bien que n'étant pas à l'origine de ces documents, les reconnaît pour valables (en cas de litiges, par exemple). Ses représentants administratifs et techniques, en concertation avec les notables, fixent les règles de gestion (dates d'entrée et de sortie). Les propriétaires coutumiers prennent les décisions locales (autorisation de pâture pour un troupeau étranger, autorisation de fauche).

En 1985, il n'y a plus de bourgoutières dans le Cercle de Tombouctou du fait de l'aléa climatique.

Pendant la phase d'exécution du projet (1985-1987), des Comités de gestion des bourgoutières sont mis en place dans chaque collectivité. Ils comprennent des représentants de la collectivité, un représentant du service de l'Elevage, un représentant de VSF (ces deux derniers en 
tant que conseillers). Le rôle de l'administration durant cette période est d'autoriser les régénérations et d'intervenir en cas de litiges de terres.

Depuis 1987, la situation est la suivante:

- la durée de vie des Comités de gestion n'a pas dépassé la période d'implication directe de VSF dans les activités de régénération;

- l'Etat intervient en cas de problème foncier, mais non dans la gestion des bourgoutières ;

- les décisions de cet ordre sont prises au sein des collectivités.

II n'y a donc plus d'intervention administrative directe et centralisée pour l'exploitation des anciennes bourgoutières et aucune autre structure moderne ne s'est mise en place autour des bourgoutières régénérées. Parmi les explications possibles, on peut citer :

- l'absence d'intrants ou de technologies complexes ;

- le retrait de l'Etat :

- la volonté du pouvoir traditionnel de garder la maîtrise sur les deux volets majeurs : le foncier et la gestion.

A l'inverse, la recrudescence de problèmes fonciers autour des zones régénérées aurait pu être le ciment pour créer de nouvelles structures d'organisation. Cette absence n'a cependant pas gêné le processus de régénération. Aussi tant que le pouvoir traditionnel est solide et raisonnablement démocratique, et que les activités n'impliquent pas de technologie complexe, il est inutile de vouloir superposer de nouveaux centres de décisions.

\section{Répartition des terres}

\section{Au niveau d'une collectivité}

Avant la période de régénération, la bourgoutière avait un statut de pâturage naturel, donné par Dieu aux hommes. La propriété et l'exploitation étaient collectives, bien que, en réalité, les décisions fussent prises par un nombre réduit de personnes. La seule exploitation individuelle était la fauche, réalisée sur des surfaces réduites et soumise à autorisation par l'autorité traditionnelle. L'organisation des activités de régénération a nécessité une parcellisation des surfaces effectuée par le pouvoir traditionnel. La parcellisation et le travail de régénération ont eu des conséquences capitales :

- la bourgoutière a changé de statut ; de pâturage naturel, elle est devenue un champ de culture $(1,6)$;

- le travail individuel sur les parcelles a généré le sentiment d'un droit d'usufruit.

Ces modifications ont eu lieu aussi bien chez les sédentaires que chez les nomades.

\section{Au niveau général}

Le programme de régénération a donné lieu à des répartitions nouvelles de terres entre les collectivités. Celles-ci ont eu lieu en présence des différentes parties et de l'administration. La seule trace écrite de ce travail est l'inscription des plaines à régénérer (avec la collectivité correspondante) dans la programmation du Comité local de développement. En pratique, l'impact de ces répartitions doit être modulé :

- certaines se sont transformées en bail à durée limitée (cinq ans) ;

- dans d'autres cas, le pouvoir traditionnel a feint, devant l'administration, d'accepter le partage mais celui-ci n'a pas eu lieu ou a été modifié (surface moins grande, plaine moins productive, etc.). Ces exemples montrent bien la permanence du pouvoir traditionnel.

\section{Gestion des terres}

L'évolution la plus notable concerne les pratiques d'exploitation avec la systématisation quasi totale de la fauche. La présence de bottes de foin sur chaque toit de concession ou dans chaque arbre proche d'une tente dans un campement nomade, montre qu'un vrai droit d'usufruit a bien été accordé à chaque exploitant, les cas de métayage étant rares.

La plupart des décisions sont donc prises collectivement et sous l'égide du pouvoir traditionnel : creusement d'un canal pour accélérer et maîtriser l'entrée de l'eau, édification d'une digue, entrée et retrait des animaux, organisation de travaux d'extension. Ces différents exemples montrent la nécessité d'une institution convaincue, écoutée et surtout capable de faire appliquer les décisions. Dans la majorité des cas, les dégradations observées entre 1988 et 1992 peuvent être rapportées soit à des conditions climatiques particulières sur un site, soit à la faiblesse de cette institution.

Quant aux décisions de fauchage et de travail de régénération (repiquage après une mauvaise année), elles sont prises au niveau individuel et ces tâches sont généralement bien et régulièrement effectuées. L'attribution individuelle de parcelles offre donc la garantie d'un bon entretien et d'une bonne exploitation dans le cadre des règles collectivement fixées (1)

\section{Commercialisation}

Au cours de la période 1985-1987, certaines collectivités impliquées dans le programme de régénération ont eu des effectifs de bétail très réduits, d'où un surplus de fourrage. Deux systèmes de valorisation de ce surplus ont été observés :

- location d'une partie de la bourgoutière à un troupeau étranger ;

- vente du fourrage sur le marché de Tombouctou.

Cette deuxième modalité ne s'est' pas démentie depuis puisque d'une part, la période de vente du fourrage cor- 
respond à la période de soudure où tout revenu monétaire prend une grande valeur et d'autre part, le prix obtenu est intéressant, le revenu à l'hectare étant deux fois supérieur à celui dégagé par la pâture directe.

La filière de commercialisation associe des producteurs, des piroguiers qui transportent le bourgou depuis les sites éloignés, des âniers qui font les derniers kilomètres jusqu'à Tombouctou, des vendeurs, des commerçants qui font crédit par avance sur récolte.

En conclusion, cette filière s'est créée sans aide, montrant que lorsqu'un marché existe, l'ensemble des opérateurs a la capacité de s'organiser.

\section{Autres initiatives}

Depuis la période 1970-1985, les activités humaines se concentrent petit à petit sur la frange fluviale. Les agriculteurs Songhaï, maintenant propriétaires de bétail, côtoient les éleveurs Tamacheq et Maure qui diversifient leurs activités. Chacun de ces groupes évolue vers un type commun d'agro-éleveur qui associe la riziculture irriguée et l'élevage plus ou moins transhumant en fonction des conditions climatiques.

Par ailleurs, on constate l'apparition d'initiatives individuelles, concrétisées sur le plan foncier par une concession à l'écart du village où le promoteur mène des activités intégrées d'agriculture et d'élevage. Cette évolution, à côté des structures collectives classiques, préfigure peutêtre la manière dont la production sera organisée à l'avenir sur la frange fluviale du Niger, du moins au Mali.

\section{Domaine du foncier}

\section{Activités de régénération. et droits fonciers traditionnel et moderne}

Lorsque les possibilités de règlement direct de litiges pour l'usage de la terre entre les pouvoirs traditionnels échouent, ceux-ci font appel à l'arbitrage de l'Etat (voir encadré). Mais celui-ci n'assume pas cette responsabilité et ne tranche pas, il renvoie les pouvoirs traditionnels dos à dos, perdant l'opportunité de jouer un rôle moteur dans l'organisation foncière. En l'absence de clarté de la part de l'Etat, la règle coutumière qui prévaut le plus souvent est celle de la mise en valeur, édictée par les populations d'agriculteurs et défavorable aux activités d'élevage. Les seuls documents de l'administration ayant accompagné le programme de régénération sont les inscriptions dans les programmations du Comité local de développement. Ces documents ont valeur d'autorisation de régénérer, mais restent dépourvus de toute valeur foncière, au sens juridique. Cette situation deviendra de plus en plus difficile à gérer avec le temps :

- la concentration des activités sur la frange fluviale va entraîner une augmentation des conflits ;
- les promoteurs d'activités de régénération risquent de se décourager en l'absence de garanties de sécurité foncière reconnues valables pour tous ;

- à l'inverse, des pseudo-activités de régénération risquent d'éclore un peu partout, dans un but de marquage du territoire.

Un statut pour les zones régénérées est nécessaire pour limiter ces inconvénients. II doit faire référence à des données techniques (critères de zones régénérées) et à des données foncières (droit d'usage, propriété). La reconnaissance d'une propriété collective gérée' par le pouvoir traditionnel semble la solution la plus simple pour surmonter les contradictions actuelles. Néanmoins, l'apparition d'initiatives personnelles prometteuses doit aussi être encouragée. Une possibilité d'appropriation individuelle doit donc aussi exister.

\section{Régénération et nomadisme}

Le mode de relation à la terre est traditionnellement différent chez les nomades et chez les agriculteurs. Historiquement, les nomades ne se sentent pas propriétaires de la terre, dont il ne font que prélever la production sans la travailler. Comme le montre l'exemple du conflit rapporté, ce temps semble révolu. Le travail de la terre pour la régénération a développé le sentiment de propriété foncière. En corollaire, la nécessité d'un entretien des zones régénérées a sans conteste un effet de sédentarisation des groupes nomades.

\section{CONCLUSION}

Le programme de régénération des bourgoutières dans le Cercle de Tombouctou au Mali est un exemple de lutte, localement réussi, contre la dégradation des ressources naturelles. La capacité de la population à entretenir et à augmenter les surfaces initialement régénérées en est la preuve. Plusieurs facteurs de réussite sont réunis : le programme répond à une demande effective, la plus-value apportée par l'activité est forte, l'activité n'est pas exigeante en temps de travail, enfin la technique est simple et sans intrants.

Dans la majorité des cas, le pouvoir traditionnel a été l'interlocuteur principal. II est difficilement contournable, tant cette action est liée aux traditions et aux règles foncières qui en découlent. Mais d'autres opérateurs individuels émergent maintenant et doivent être considérés aussi comme des partenaires potentiels qu'il faudra intégrer à part entière. Quant aux facteurs de risque qui menacent ces régénérations, ce sont:

- la possibilité d'une nouvelle dégradation climatique ; - un manque de sécurisation foncière qui fragilise les résultats.

La création d'un statut pour les zones régénérées est donc indispensable à la pérennité et à l'amplification de cette ressource naturelle. 


\begin{tabular}{|c|}
\hline 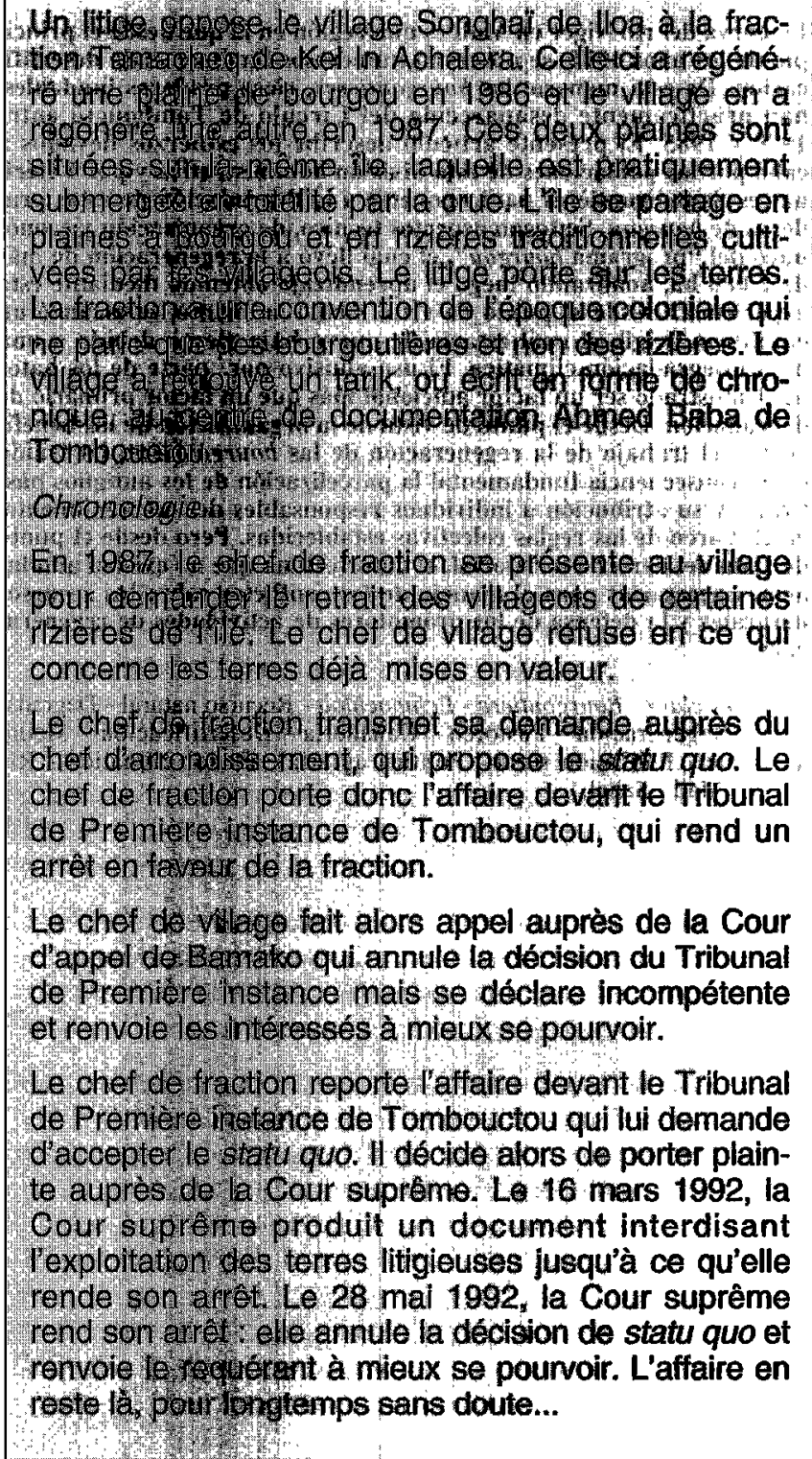 \\
\hline
\end{tabular}

\section{REMERCIEMENTS}

L'auteur tient à exprimer sa gratitude à tous ceux, Maliens et étrangers, qui l'ont aidé dans la réalisation de sa tâche. II remercie également les lecteurs scientifiques et la rédaction de la Revue pour leurs conseils et leur aide dans la mise en forme du texte.

\section{BIBLIOGRAPHIE}

1. BONIS CHARANCLE (J.M.). Fiche d'expérience 7 : régénération des bourgoutières à Tombouctou. In : ROCHETTE (R.M.). Le Sahel en lutte contre la désertification, leçons d'expériences. Weikersheim, Deutschland, GTZ, Verlag Joseph Margraf, 1989. p. 115-133.

2. BOUDET (G). Contribution au contrôle continu des pâturages tropicaux en Afrique occidentale. Revue Élev. Méd. vét. Pays trop., 1977, 30 (4) : $387-406$.

3. DULIEU (D). Les bourgoutières du fleuve Niger, contraintes, possibilités d'action. République du Niger, étude thématique. Maisons-Alfort, IEMVT, 1989. p. 8.

4. FRANCOIS (J.), RIVAS (A.), COMPERE (R.). Le pâturage semi-aquatique à Echinochloa stagnina (Retz) P. Beauv. Etude approfondie de la plante bourgou et des bourgoutières situées en zone lacustre au Mali. Bull. Rech. agron. Gembloux, 1989, 24 (2) : 163.

5. LAINE (F.). Un programme Vétérinaires Sans Frontières : la régénération de "bourgoutières" dans le cercle de Tombouctou (bilan après 2 ans). Thése Doct. Vét., Ecole Nationale Vétérinaire de Lyon, 1987. p. 24.

6. MERCAT (L.), PINGET (K.), VAN DER HEYDEN (D.). Impacts socio-économiques de la régénération des bourgoutières dans le cercle de Tombouctou au Mali. Etude socio-économique de I'ISARA. Lyon, ISARA, 1990. p. 15.

7. SEGUIN (A). Contribution à l'étude du bourgou (Echinochloa stagnina). Thèse Doct. Vét., Ecole Nationale Vétérinaire de Nantes, 1986. $109 \mathrm{p}$.

8. VSF. Rapports d'activité VSF-Tombouctou de 1987 à 1992. Lyon, VS.F. 


\section{J.-M. Bonis Charancle}

BONIS CHARANCLE (J.-M.). Natural resources management : regeneration of bourgoutières in the bend of the Niger in Mali. Revue Élev. Méd. vét. Pays trop., 1994, 47 (4): 425-434

Since the early $1970 \mathrm{~s}$, the desertification process in the bend of the Niger river in Mali has progressed rapidly. The once abundant natural flood-plain grassland of bourgou virtually disappeared between 1970 and 1985 in the Timbuktoo region. The article describes the efforts of the local population and Vétérinaires Sans Frontières to stop the degradation of bourgoutières. While presenting the technical, organisational and economic aspects of the "bourgou program", the author also analyses program results and lessons learned that led to the regeneration of over 2,000 hectares of bourgou pastures. A fragile ecological balance has been reached that could be broken by a renewed dry period. Livestock activities are perceived as an additional factor in the desertification process rather than a primary cause. The regeneration of bourgoutières has had significant effects on landuse systems. Bourgou pastures are now divided into separate plots where responsibility for their maintenance lies with an individual within the framework of collective land-use rules. The author suggests that the instruments necessary for the resolution of natural resource management conflicts and the defense of those involved in regeneration activities are not yet in place.

Key words: Bourgoutière - Echinochloa - Natural resource Degradation - Regeneration - Grazing - Animal husbandry - Desertification - Environment - Rural population - Land policy - Climatic factor Niger river - Mali - Sahel.
BONIS CHARANCLE (J.-M.). Manejo de los recursos naturales : regeneración de bourgoutières (Echinochloa spp.) en la curva del río Niger en Mali. Revue Élev. Méd. vét. Pays trop., 1994, 47 (4) : 425-434

La curva del rió Niger en Mali es una región en la que desde el principio de los años 1970, se observan numerosos fenómenos de desertificación. De esta manera, las bourgoutières, ricas praderas inndables, han practicamente desaparecido del Círculo de Tombouctu entre 1970 y 1985. El presente artículo describe los esfuerzos llevados a cabo por las poblaciones de esta división administrativa y por Vétérinaires Sans Frontières para oponerse a la degradación de estas praderas de bourgou. Se tocan aspectos técnico, de organización y económico del "programa bourgou", el cuál llevó a la regeneración de más de 2000 ha, analizando luego el aprendizaje obtenido mediante esta experienca. Desde el punto de vista medio ambiente, se alcanźó un equilibrio frágil, el cuál estaría de nuevo bajo riesgo debido a una nueva degradación climática. El uso abusivo por parte de los hatos ha demostrado ser un factor adicional más que un factor primario de degradación. Desde el punto de vista de la organización de los beneficiaros, el trabajo de la regeneración de las bourgoutières ha traído como consecuencia fundamental la parcelización de los antiguos pastizales y su atribución a individuos responsables del mantenimiento, en el marco de las reglas colectivas establecidas. Pero desde el punto de vista del derecho territorial, el autor estima que no existen aún las armas necesarias para el manejo de los conflictos sobre los recursos naturales y la defensa de los promotores de actividades de regeneración.

Pulabres clave : Bourgoutière - Echinochlou - Recurso natural - Degradación - Regeneración - Pastoreo - Ganadería - Desertificación - Medio ambiente - Población rural - Política agraria - Factor climático - Río Nigcr - Mali - Sahcl. 